\title{
La bibliothèque conventuelle selon l'ordre des prêcheurs
}

\section{Frère Rémy Valléjo}

\section{(2) OpenEdition}

1 Journals

Édition électronique

URL : http://journals.openedition.org/rbnu/924

DOI : $10.4000 /$ rbnu.924

ISSN : 2679-6104

\section{Éditeur}

Bibliothèque nationale et universitaire de Strasbourg

\section{Édition imprimée}

Date de publication : 1 mai 2017

Pagination : $74-81$

ISBN : 9782859230661

ISSN : 2109-2761

\section{Référence électronique}

Frère Rémy Valléjo, "La bibliothèque conventuelle selon l'ordre des prêcheurs », La Revue de la BNU [En ligne], 15 | 2017, mis en ligne le 01 novembre 2019, consulté le 15 décembre 2020. URL : http:// journals.openedition.org/rbnu/924 ; DOI : https://doi.org/10.4000/rbnu.924

\section{(c) (i) (2)}

La Revue de la BNU est mise à disposition selon les termes de la Licence Creative Commons Attribution - Pas d'Utilisation Commerciale - Partage dans les Mêmes Conditions 4.0 International. 


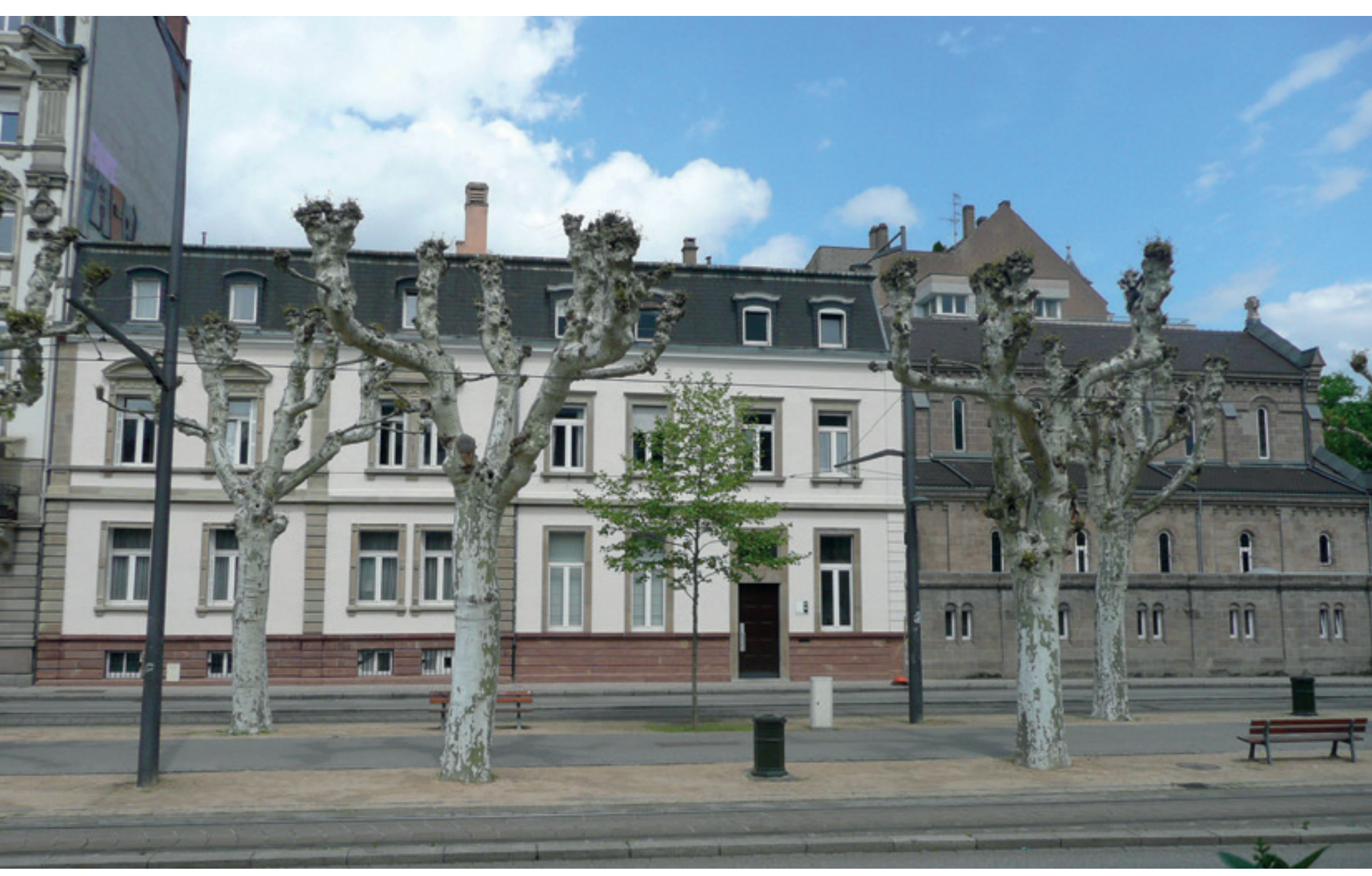

Vue générale du couvent

des dominicains de Strasbourg 


\section{LA BIBLIOTHÈQUE CONVENTUELLE SELON L'ORDRE DES PRÊCHEURS \\ PAR LE FRÈRE RÉMY VALLÉJO O. P.}

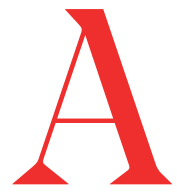

$\mathrm{u} 21^{\mathrm{e}}$ siècle, et ce depuis la fondation de l'ordre des prêcheurs en 1215, la bibliothèque d'un couvent dominicain répond aux exigences d'une vocation singulière dans le paysage des institutions religieuses d'Occident. En effet, il ne s'agit pas d'une simple bibliothèque monastique consacrée à l'approfondissement de la foi et du mystère chrétien selon les usages de la « lectio divina ", mais d'une bibliothèque conventuelle destinée à l'étude pour l'intelligence de l'homme, du monde et du mystère divin dans une dimension universaliste. Inscrite au cœur de la vie conventuelle, dans sa dimension communautaire et contemplative, elle est un outil essentiel de la mission des frères prêcheurs députés par vocation à l'évangélisation de la parole de Dieu. "Contemplare et contemplata aliis tradere": selon un adage qui depuis le Moyen Âge fait autorité dans l'ordre des prêcheurs, tout frère, député par vocation à l'évangélisation de la Parole, est appelé à « contempler et à transmettre ce qu'il a contemplé ». De nos jours, la bibliothèque d'un couvent dominicain ne ressemble en rien à l'antique et mythique « libraria » du couvent Saint-Jacques qui, au Moyen Âge, est un foyer de la révolution du savoir scolastique au sein de l'Université de Paris. Cependant, aussi modeste soit-elle, elle doit répondre aux exigences intellectuelles que suscite un monde sécularisé, post-moderne et pluri-religieux. En effet, qu'il s'agisse d'une simple réalité conventuelle en France, ou d'une institution comme l'Institut dominicain des études orientales au Caire, la bibliothèque d'un couvent dominicain tente d'offrir à ses lecteurs les moyens de comprendre un monde en pleine mutation.

\section{Le propos de l'ordre des prêcheurs}

L'ordre des prêcheurs est fondé à Toulouse en 1215 par saint Dominique, sous la protection de l'évêque Foulque, et confirmé à Rome le 22 décembre 1216 par le pape Honorius III. Appelés à vivre les exigences de la vie conventuelle, les frères dominicains sont, selon leurs Constitutions, totalement députés à l'évangélisation de la parole de Dieu. "Athlètes de la foi », selon une expression que la tradition prête au pape Honorius III, ils prêchent « verbo et exemplo », par la parole et par l'exemple, toujours en quête d'une intelligence de la foi dans un monde en perpétuelles transformations.

Engagés dans cette prédication «verbo et exemplo », les frères dominicains sont appelés à répondre aux exigences de la communion fraternelle, telle que l'exprime saint Augustin dans le préambule de sa règle inspirée par la première épître de saint Jean : «Avant tout, frères très chers, aimons Dieu, aimons le prochain, ce sont les commandements qui nous sont donnés en premier ». À cette fin, la règle recommande aux frères de vivre " ensemble dans l'unanimité, ne faisant qu'un cœur et qu'une âme en Dieu », en mettant tous leurs biens en commun. Si l'unanimité, comme signe visible de la communion en Dieu, est au cœur de la vie dominicaine, l'étude pour le service du prochain en est la fin, et tout dans la vie du frère doit y concourir. Ainsi, selon les Constitutions primitives, rédigées lors du chapitre général de Paris en 1228, le supérieur a « en son couvent pouvoir de dispenser les frères chaque fois qu'il l'estimera convenable, principalement en ce qui paraîtrait faire obstacle à l'étude, à la prédication, ou au bien des âmes, puisque l'on sait que notre ordre, dès les 
débuts, a spécialement été institué pour la prédication et le salut des âmes et que notre étude doit tendre par principe, avec ardeur et de toutes nos forces, à nous rendre capables d'être utiles à l'âme du prochain ${ }^{1}$.

Conformément aux Constitutions primitives, l'ordre des prêcheurs est institué « pour la prédication et le salut des âmes ». Les frères doivent « aller et se comporter partout en toute perfection et esprit religieux, comme des hommes évangéliques suivant les traces de leur sauveur, parlant avec Dieu ou de Dieu, en eux-mêmes ou avec le prochain ". Saint Dominique, dont aucun sermon n'est conservé, préconise alors une vie religieuse et une formation qui, par leur genre et leur qualité, préparent directement à la prédication. L'ordre devient alors une véritable école de prédication où s'illustrent très tôt Jourdain de Saxe, Raymond de Pennafort, Jean le Teutonique, Humbert de Romans et Jean de Verceil.

La prédication est l'œuvre d'une contemplation. En effet, conformément à une expression de la tradition dominicaine déjà citée (« contemplare et contemplata aliis tradere »), l'art de prêcher nécessite de contempler pour transmettre ce qui a été contemplé. Tout entière consacrée à la prédication, la vie dominicaine se ressaisit dans le propos de Thomas d'Aquin qui dans sa Somme théologique (II ${ }^{\mathrm{a}} \mathrm{II}^{\mathrm{ae}}, 188$ ), affirme qu'une " vie active dans laquelle quelqu'un transmet aux autres les fruits de ses contemplations en prêchant et en enseignant, est plus parfaite qu'une vie livrée uniquement à la contemplation, car une telle vie présuppose une grande richesse contemplative $»$.

Pendant huit siècles, la prédication des frères prêcheurs ne cesse d'évoluer dans ses modes d'expression. Née au $13^{\text {e }}$ siècle dans un contexte de " disputatio ", au sens médiéval et universitaire d'une question disputée, elle se raidit au $16^{\mathrm{e}}$ siècle, avec la controverse entre Réforme et contre-réforme, avant de s'engager dans un dialogue prôné par le concile de Vatican II au $20^{\mathrm{e}}$ siècle. En déterminant le rapport des frères à la prédication, la "disputatio ", la controverse et le dialogue ont une influence directe sur l'étude et par conséquent sur la manière de concevoir les bibliothèques conventuelles.

\section{des prêcheurs \\ La bibliothèque selon les Constitutions}

Depuis le chapitre général de Paris, en 1228, l'étude représente un des points essentiels des constitutions de l'ordre des prêcheurs qui, à maintes reprises, préconise plusieurs dispositions pour l'existence et la gestion d'une bibliothèque au sein d'un couvent dominicain. Selon le texte même des Constitutions primitives, « notre étude doit viser principalement, ardemment et avec le plus grand soin, à ce que nous puissions être utiles à l'âme du prochain ».

Jusqu'à nos jours, les chapitres généraux qui régissent la vie et la mission des frères de l'ordre dominicain ne cessent de revenir sur les questions relatives à l'étude pour répondre aux exigences de la prédication. En effet, dans le $\mathrm{n}^{\circ} 87$ des Constitutions de l'ordre des prêcheurs, « les supérieurs feront grand cas de l'étude et la développeront intensément, en veillant à ce que tous les frères aient les moyens et les possibilités d'étudier ». Parmi ces moyens destinés à l'étude, la bibliothèque relève d'un statut particulier puisque son administration et son utilisation exigent des dispositions qui sont formellement inscrites dans les constitutions, telle l'allocation d'une somme sur le budget annuel d'un couvent. L'acquisition d'ouvrages n'est donc pas envisagée comme un fait aléatoire ou surérogatoire, mais comme constitutive de la réalité conventuelle. Ainsi, selon le $\mathrm{n}^{\circ} 88$, « il revient principalement au supérieur du couvent de veiller à ce que la bibliothèque soit pourvue des livres nécessaires et qu'une somme suffisante chaque année soit allouée pour son développement ». Responsable $\mathrm{du}$ fonds de la bibliothèque, avec son spectre des sciences bibliques, théologiques, philosophiques et historiques, le bibliothécaire est aidé dans sa tâche par le lecteur conventuel qui, selon le $\mathrm{n}^{\circ} 326$ bis « doit veiller à ce que la bibliothèque du couvent soit mise à jour et enrichie des livres nécessaires ". Au Moyen Âge, l'enseignant est un "lecteur " (lector) parce que son enseignement est à base de lecture et de commentaires de textes. Aujourd'hui, le titre demeure appliqué à tout frère reconnu pour ses compétences intellectuelles et universitaires, ouvert à l'actualité des questions théologiques et ouvert sur tous les domaines de recherche. Conseiller privilégié du bibliothécaire, 


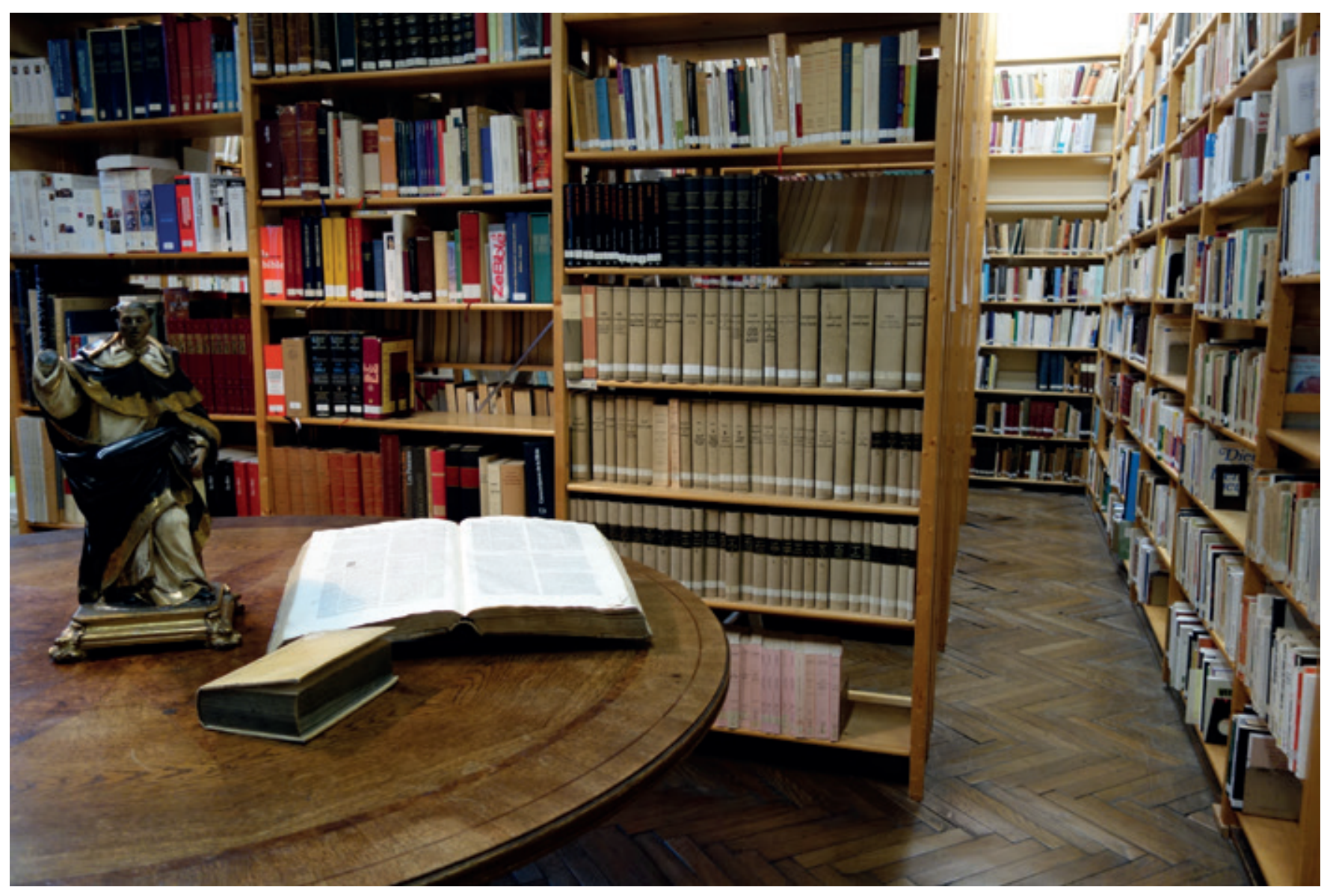

La bibliothèque du couvent des dominicains de Strasbourg 
le lecteur l'est tout autant du prieur, ou supérieur, $\mathrm{du}$ couvent. En effet, selon le $\mathrm{n}^{\circ} 88$, « il y aura un lecteur conventuel des études qui aidera le supérieur à développer la vie intellectuelle de la communauté, à moins que le supérieur ne s'acquitte lui-même de cette tâche ". Depuis le $13^{\text {e }}$ siècle, au temps où la bibliothèque conventuelle est un lieu d'exception tant par le savoir et la doctrine dont elle est le dépôt que par le coût financier qu'elle représente, les charges de bibliothécaire et de lecteur conventuel sont toujours demeurées dans l'ordre dominicain de véritables offices institués, avec même une approbation du provincial pour le lecteur conventuel. $\mathrm{Si}$, dans les contextes troublés de la contre-réforme et de la crise moderniste, ces charges sont confiées à des officiers garants de la doctrine, de nos jours elles échoient à des frères reconnus non seulement pour leur compétence, mais aussi pour leur capacité à s'ouvrir aux questions du monde et de la société. Ainsi, selon le $\mathrm{n}^{\circ} 330$, «le prieur doit instituer un bibliothécaire, un lecteur conventuel [...] Pour chacun d'eux le chapitre provincial doit déterminer les conditions, la durée, les responsabilités et les autres points opportuns ». Certes, la bibliothèque d'un couvent dominicain n'est plus de nos jours ce lieu d'exception qu'Umberto Eco met en scène dans Le Nom de la rose, mais elle demeure le lieu emblématique de l'étude à laquelle se vouent les frères prêcheurs.

Pour parfaire la formation et la mission des frères, les Constitutions de l'ordre des prêcheurs recommandent dans le $\mathrm{n}^{\circ} 91$ la création et la gestion de centres d'études. En effet, «bien que dans chaque couvent la vie intellectuelle doive être florissante, il doit $\mathrm{y}$ avoir cependant des centres où les frères se consacrent plus particulièrement à l'étude ». Dans l'ordre des prêcheurs, la bibliothèque ne relève pas de la seule structure conventuelle mais aussi de ce qui, selon les Constitutions, est appelé un centre d'études. D'après le $\mathrm{n}^{\circ} 91$, « un centre d'études dans l'ordre est une communauté de frères qui à temps plein et d'une façon stable s'adonne à l'étude ; elle se compose d'au moins trois frères, doués des qualifications requises ; elle doit être dotée d'une bibliothèque en rapport avec ces études et de tous autres instruments de travail, sans parler des ressources économiques stables ». Cette institution, avec la bibliothèque conventuelle qui lui est attachée, participe non seulement de la vie intellectuelle des frères, mais aussi et surtout de leur mission. Parmi les centres d'études les plus connus de l'ordre des prêcheurs, l'École biblique et archéologique française ${ }^{2}$ de Jérusalem, fondée en 1890 , et l'Institut dominicain des études orientales ${ }^{3}$ du Caire, créé en 1953, occupent une place particulière dans le monde des sciences bibliques et du dialogue interreligieux, non seulement au sein de l'Église catholique, mais aussi et surtout dans le monde de la recherche.

\section{La bibliothèque d'un couvent de frères prêcheurs}

Conformément aux Constitutions et aux orientations votées et adoptées par les frères prêcheurs lors de leurs chapitres généraux, la bibliothèque d'un couvent de dominicains est un outil essentiel à la prédication. Dès lors, en offrant une intelligence du monde et du mystère divin, grâce aux ouvrages qui s'y trouvent rassemblés, elle répond aux exigences théologiques et pastorales de cette prédication.

"Fides quaerens intellectum ": selon une expression traditionnelle prêtée à Anselme de Cantorbéry qui, au $12^{\mathrm{e}}$ siècle, reprend une pensée déjà longuement développée par Augustin, évêque d'Hippone, la théologie est « la foi en quête d'intelligence ». Tout au long de son histoire, en quête d'une intelligence de la foi chrétienne, la théologie se déploie en un ensemble de disciplines corrélées et hiérarchisées les unes par rapport aux autres. Ce déploiement, avec ses évolutions successives, telles la définition des "lieux " théologiques par le dominicain Melchior Cano au $16^{\mathrm{e}}$ siècle et les orientations de l'école dominicaine de théologie du Saulchoir au $20^{\text {e }}$, détermine la réalité d'une bibliothèque conventuelle.

Conformément à la hiérarchisation formalisée par Melchior Cano, le premier « lieu » théologique fondamental est constitué par les sciences bibliques, telles l'exégèse de l'Ancien et du Nouveau Testament, la théologie biblique et l'herméneutique. Si, selon l'expression du pape Léon XIII, la Bible est « l'âme de toute la théologie ", elle exige plusieurs types d'études attentives et raisonnées pour éviter les écueils de toute lecture fondamentaliste. Une bibliothèque se doit de rassembler les textes sacrés en hébreu, en grec et en 
Constitutions de l'ordre des prêcheurs

(édition de Rome, 1690 ; coll. Bibliothèque des dominicains de Strasbourg)
Jean Capraeolus, Livre de la défense de la théologie du divin docteur Thomas d'Aquin,

Venise, 1483 (coll. Bibliothèque du Saulchoir)

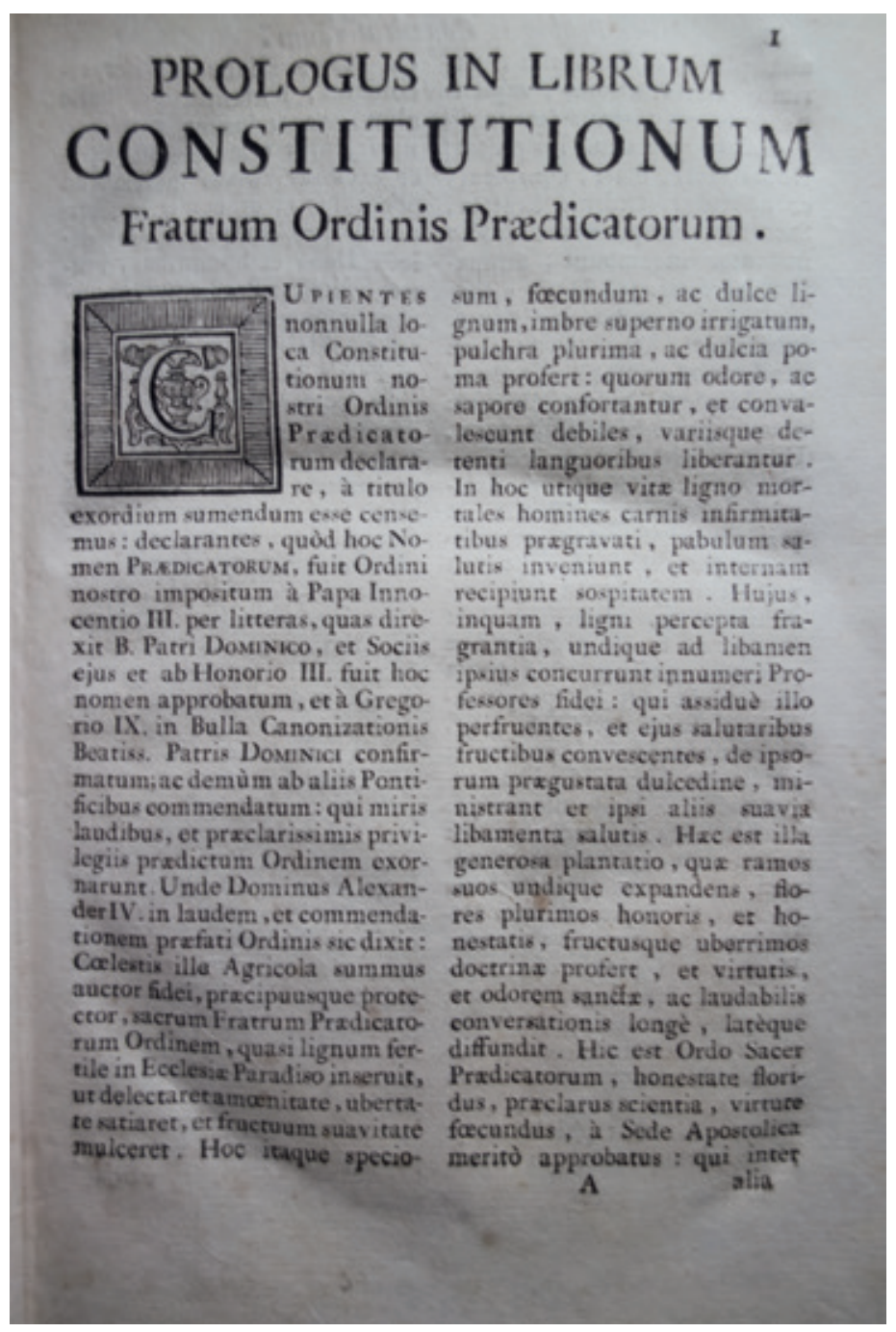

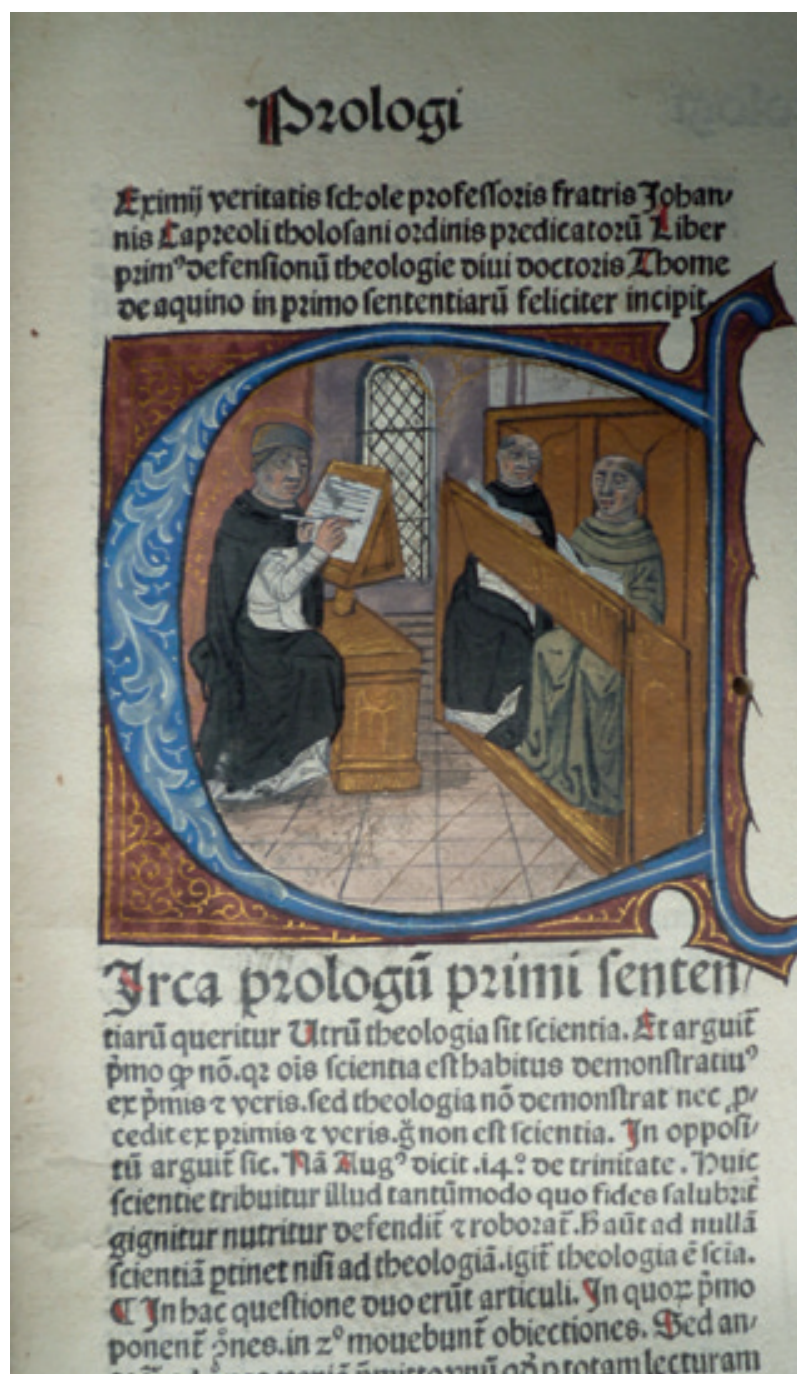


latin, ainsi que leurs traductions respectives, mais aussi les commentaires et les ouvrages d'exégèse. Le travail du frère Marie-Joseph Lagrange (18551938) et des frères de l'École biblique à Jérusalem a profondément bouleversé les rayonnages des bibliothèques dominicaines où abondent désormais les ouvrages d'exégèse historico-critique et d'analyse littéraire. Aujourd'hui encore, l'EBAF poursuit son œuvre avec la «Bible en ses traditions » (BEST), qui envisage le texte biblique dans toutes ses composantes exégétiques, patristiques et artistiques, œcuméniques et interreligieuses. Ce labeur pluridisciplinaire, que seule l'arborescence numérique peut aujourd'hui raisonnablement déployer, oblige ainsi les bibliothèques conventuelles dominicaines à s'adapter aux usages des nouvelles technologies.

L'usage du corpus patristique exige lui aussi de recourir au mode numérique. Parmi les premiers commentaires des Écritures, les textes patristiques ont une place particulière. En effet, ils participent, dès les premiers siècles de l'Église, de cette tradition sans laquelle toute lecture littérale de la Bible peut engendrer de dangereux fondamentalismes. Les collections de textes patristiques grecs, latins et orientaux, telle la célèbre Patrologiae cursus completus éditée par JacquesPaul Migne, ou les plus récentes Sources chrétiennes, constituent donc un deuxième "lieu " fondamental de la théologie. L'accès numérique de ce corpus, auquel s'ajoute un impressionnant fonds patristique oriental en cours d'édition, représente désormais un outil de recherche incontournable pour une meilleure connaissance des sources théologiques des Églises d'Orient et d'Occident. Par ailleurs, comme en témoigne la récente actualité du Proche-Orient, avec l'exode des chrétiens orientaux, puis la destruction en 2015 des bibliothèques de Mossoul par les armées de Daech, il est nécessaire qu'une bibliothèque conventuelle offre aux frères dominicains orientaux expatriés les moyens de demeurer enracinés dans leur propre tradition.

Comme l'affirme le frère dominicain MarieDominique Chenu (1895-1990), pionnier en 1937 de l'école de théologie du Saulchoir, "l'intelligence du mystère n'est perceptible que dans l'histoire où elle se déroule, dans une histoire sainte. C'est évidemment aller à l'encontre d'une conception intemporelle de la théologie, savoir immobile à travers le temps et l'espace. La voici, la théologie, entraînée dans le relativisme, c'est-à-dire dans le jeu complexe des relations qui modifient continuellement non plus, bien sûr, le contenu radical de la foi, mais ses expressions ». Dès lors, les sciences historiques, non seulement l'histoire de l'Église, mais aussi la réflexion théologique sur l'Histoire, sont de véritables instances de discernement dans une ère tentée par le fondamentalisme.

Depuis Justin le Philosophe (mort en 165), la philosophie tient une place essentielle dans les études de théologie. Les ouvrages des présocratiques, de Socrate, de Platon, d'Aristote et des néoplatoniciens, des médiévaux, des modernes et des contemporains sont autant d'approches philosophiques qui permettent d'envisager le mystère de l'homme sans lequel le mystère de l'Incarnation est difficilement envisageable. Depuis quelques décennies, la philosophie d'Outre-atlantique, illustrée notamment par l'œuvre de Charles Taylor, offre des outils de réflexion que les frères dominicains, longtemps formés par les philosophies française et allemande, ne peuvent plus désormais négliger. Le bibliothécaire et le lecteur conventuel sont donc tous deux obligés d'opérer un déplacement dans leur politique d'acquisition afin d'engager leurs propres frères dominicains dans de nouveaux paradigmes philosophiques.

Dans l'ordre des prêcheurs, la théologie est traditionnellement envisagée à l'aune de l'œuvre et de la pensée de Thomas d'Aquin (1204-1274). Selon le $\mathrm{n}^{\circ} 82$ des Constitutions, «le meilleur maître et modèle » de l'accomplissement de la tâche théologique « est saint Thomas dont l'Église recommande particulièrement la doctrine reçue par l'ordre comme un patrimoine exerçant une influence féconde sur la vie intellectuelle des frères et lui conférant un caractère propre ». Si l'œuvre de l'Aquinate est révolutionnaire au $13^{\mathrm{e}}$ siècle, d'autres révolutions ont été opérées dans le domaine théologique jusqu'au seuil du $21^{\mathrm{e}}$ siècle. Loin de se réduire à la seule Summa theologica de Thomas d'Aquin, la théologie systématique se déploie alors en plusieurs approches où la théologie fondamentale, la théologie dogmatique, la théologie morale et l'éthique reçoivent une coloration particulière. Dans une perspective dialogale encouragée par l'œuvre du frère Yves Congar (1904-1995), mais aussi et surtout par le concile Vatican II, la théologie catholique y est envisagée à l'aune de l'œcuménisme et du dialogue interreligieux, où sont convoquées les 


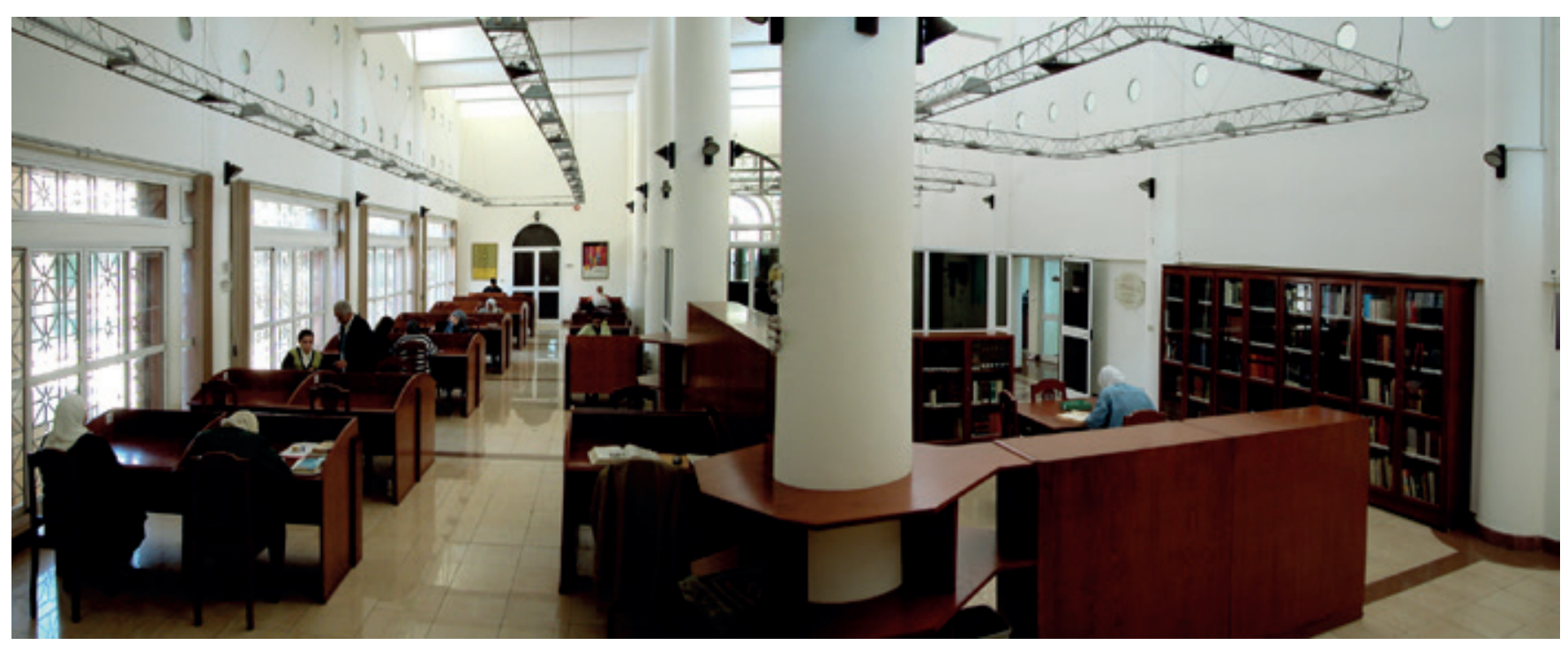

Vue générale de la bibliothèque de l'Institut dominicain des études orientales au couvent des dominicains du Caire

différentes confessions chrétiennes, mais aussi les traditions monothéistes du judaïsme et de l'islam, ainsi que les sagesses d'Orient. Toutefois, ce dialogue ne concerne pas seulement les traditions religieuses, embrassant aussi, dans un monde en pleine mutation, les questions sociales, politiques, scientifiques et éthiques.

Enfin, outre la théologie pratique, la liturgie, le droit canonique, la pastorale et la pédagogie religieuse, la littérature et les arts ne sont jamais négligés, non seulement parce que ces modes d'expression expriment explicitement une expérience de foi, mais aussi et surtout parce qu'ils témoignent d'une quête intérieure qui, parfois éloignée de toute expression religieuse, dit l'essence de l'homme.

\section{Du miroir médiéval à l'arborescence contemporaine}

Miroir d'une révolution du savoir à l'époque scolastique, la bibliothèque d'un couvent de dominicains du $21^{\text {e }}$ siècle devient une arborescence des aspirations de l'homme contemporain qu'engendrent et contredisent tout à la fois les enjeux d'un monde en pleine mutation. Ayant renoncé à toute prétention encyclopédique, le bibliothécaire, aidé dans sa charge par le lecteur conventuel, tente d'honorer les questionnements qui obligent alors le frère prêcheur à trouver le langage approprié pour exprimer l'essence de son propos. Si la forme a considérablement changé, le propos de l'" ordo praedicatorum " est toujours le même et la bibliothèque demeure encore un lieu d'étude et de contemplation qui, au gré de la quête qu'il suscite, invite chaque frère dominicain à transmettre ce qu'il a contemplé.

NOTES

1- «... studium nostrum ad hoc principaliter ardenterque summo opere debeat intendere ut proximorum animabus possimus utiles esse ».

2- L'École biblique et archéologique française de Jérusalem, ou EBAF, est fondée en 1890 sous le nom d'École pratique d'études bibliques par le frère dominicain Marie-Joseph Lagrange. En 1920, elle prend son nom actuel, à la suite de sa reconnaissance comme école archéologique nationale française par l'Académie des Inscriptions et Belles-Lettres.

3- Conçu et fondé en 1928 par le frère Antonin Jaussen (1871-1962), comme un simple pied-à-terre en Égypte de l’École biblique de Jérusalem principalement destiné à l'approfondissement des sciences exégétiques, le couvent des dominicains du Caire s'inscrit dès 1937 dans le paysage universitaire et interreligieux des études orientales et des sciences islamiques. Depuis le 7 mars 1953, il abrite l'Institut dominicain d'études orientales qui se consacre aux recherches sur les sources de la civilisation arabo-musulmane dans un dialogue constant avec le corps professoral et les étudiants de l'Université al-Azhar. Pleinement engagés dans cette mission, les frères s'investissent dans la langue arabe, les sciences coraniques et la civilisation arabo-musulmane dans un constant dialogue entre paradigmes d'Orient et d'Occident. 